\title{
http://revistainvestigacionacademicasinfrontera.com
}

\section{Estudio Descriptivo de la Situación Nutricional de los Estudiantes de la Escuela Primaria Niños Héroes del Municipio de Etchojoa, Sonora}

\author{
Mtro. Jesús Alfredo Rojo Villa ${ }^{1}$ Lic. Juan Alberto Esquer Villegas ${ }^{2}$ Mtra. Marina Reyes \\ Robles $^{3}$ Mtro. Edgar Duarte Félix ${ }^{4}$
}

\begin{abstract}
Resumen
A nivel internacional se hacen cada vez más frecuentes los casos de personas con malnutrición, México no se encuentra exento de esta problemática, por un lado las tasas de obesidad siguen en aumento y a su vez, los problemas de desnutrición aquejan ya no solo a las personas en pobreza extrema. El Objetivo de la presente es el de diagnosticar el estado nutricional en los escolares de la escuela primaria Niños Héroes del Municipio de Etchojoa mediante el uso de indicadores antropométricos acordes a la edad, estudios descriptivo de corte trasversal aplicado $n=48(92.3 \%)$ de ambos sexos con rango de edad de 6 a 12 años, el muestreo es no probabilístico, los resultados obtenidos arrojan que existe una cantidad considerable de casos con problemas por déficit de nutrientes, $18.7 \%(n=9)$ presentan retraso en el crecimiento, $22.9 \%(n=11)$ presentan sobre peso y obesidad, la información obtenida es el punto de partida para la estructuración de estrategias para prevenir y combatir la problemática que aqueja ya no solo a estratos socioeconómicos altos.
\end{abstract}

Palabras clave: Estado nutricional, antropometría, escolares, antropometría

\footnotetext{
${ }^{1}$ Profesor investigador de tiempo completo en la Licenciatura en Entrenamiento Deportivo de la Universidad Estatal de Sonora, Campus Benito Juárez, estudiante del Doctorado en Ciencias del Cultura Física en la Universidad Central "Marta Abreu" de Las Villas Cuba Alfredo.rojo@Rojo.Live.com ${ }^{2}$ Licenciado en Entrenamiento Deportivo de la Universidad Estatal de Sonora Juanesquer94@ gmail.com ${ }^{3}$ Profesor investigador de Tiempo completo en la Licenciatura en Entrenamiento Deportivo de la Universidad Estatal de Sonora, Campus Benito Juárez, estudiante del Doctorado en Ciencias de la Cultura Física en la Universidad Autónoma de Nuevo León México weightmarina21 @ hotmail.com ${ }^{4}$ Profesor adjunto en la en la Licenciatura en Entrenamiento Deportivo de la Universidad Estatal de Sonora Campus Navojoa, estudiante del Doctorado en Docencia en IDESA edgarduarte_14@hotmail.com
} 


\title{
$\underline{\text { http://revistainvestigacionacademicasinfrontera.com }}$
}

\begin{abstract}
Abastract
At the international level the cases of people with malnutrition are becoming more frequent, Mexico is not exempt from this problem, on the one hand the obesity rates continue to increase and at the same time the problems of malnutrition already affect not only the people in extreme poverty. The objective of this is to diagnose the nutritional status of schoolchildren in Niños Héroes Elementary School of the Municipality of Etchojoa by using anthropometric indicators according to age, descriptive studies of transversal cut applied $n=48(92.3 \%)$ of both sexes with an age range of 6 to 12 years, the sampling is not probabilistic, the results obtained show that there is a considerable number of cases with problems due to nutrient deficit $18.7 \%(\mathrm{n}=9)$ present growth retardation, $22.9 \%(n=11)$ presented on weight and obesity, the information obtained is the starting point for the structuring of strategies to prevent and combat the problem that afflicts not only high socioeconomic strata.
\end{abstract}

Keywords: Nutritional status, anthropometry, school, anthropometry

\section{Introducción}

Las afecciones nutricionales son cada vez más frecuentes en el mundo, la globalización, la pérdida de la biodiversidad, la preferencia por los alimentos industrializados en lugar de los alimentos étnicos tradicionales y la migración del campo a la ciudad, han propiciado un cambio drástico en nuestro estilo de vida (Román, Ojeda-Granados y Panduro (2013).

El estado mexicano no se encuentra exento de esta problemática, más bien presenta una doble carga de malnutrición (Ibarra, Viveros, González \& Hernández, 2017), fenómeno alarmante ya que se cuenta con casos donde en el mismo hogar se hace presente malnutrición por déficit, especialmente en los niños y por exceso en los adultos (HurtadoQuintero et al., 2017).

La desnutrición y obesidad son enfermedades comunes ya que no se contrae por contagio, sino por factores circunstanciales y en gran parte por la desigualdad económico-social. Pese a que estas problemáticas se encuentran en los extremos de la balanza tienen un lugar importante en las estadísticas nacionales e internacionales (Farrè, 2017). 


\section{$\underline{\text { http://revistainvestigacionacademicasinfrontera.com }}$}

La desnutrición, afecta de un modo significativo a la región sur, y la obesidad, lo hace en el norte, se extienden a lo largo de todo el territorio mexicano, poniendo de manifiesto la necesidad de aumentar los esfuerzos en promover una los hábitos saludables enfatizando a la actividad física y la alimentación adecuada en todos los grupos de edad, especialmente hincapié en los niños, niñas.

En gran parte de los casos se presenta por problemas de alimentación, factor íntimamente ligado a la crisis económica, factores políticos adversos y sociales, una clara evidencia es el informe publicado por la, Organización de Naciones Unidas para la Agricultura y la Alimentación (FAO) por sus siglas en inglés, muestra que ochocientos cincuenta y cuatro millones de personas del mundo están mal alimentadas, y la mayoría de éstas se encuentran en países subdesarrollados, asimismo, estos países son los que presentan mayor retraso económico, cultural y social en el mundo (FAO, 2013).

Por lo cual, entre las premisas sociales y sanitarias se encuentran la erradicación de la pobreza y el hambre, factores que aquejan a gran parte de la población enfatizándose en las zonas serranas y rurales (Courtois, 2014).

En las edades tempranas el buen estado nutricional toma un carácter crítico como coadyuvante para el buen estado de salud, la detección precozmente del mismo y el monitoreo periódico ofrece la posibilidad de observar los cambios físicos y cognitivos que se pueden presentar ante una variación en la ingesta de nutrientes, por lo contrario es posible que la afección sea irreversible y afectar el futuro del infante.

La alimentación constituye una actividad primordial en la vida de los seres vivos, fortaleciendo el sistema inmunológico formando una barrera más fuerte contra algunas enfermedades oportunistas, de igual forma es el principal factor exógeno condicionante del crecimiento y desarrollo (Aranceta, Perèz \& Garcìa, 2002) entendiéndose como el conjunto de cambios somáticos y funcionales, que se producen desde la concepción, 


\section{$\underline{\text { http://revistainvestigacionacademicasinfrontera.com }}$}

extendiéndose por toda la vida, este proceso implica una visión dinámica, evolutiva y prospectiva en la asistencia del niño (Kliegman, Staton \& Geme, 2016).

Para que se presenten de manera idónea es imprescindible facilitar una alimentación que cubra las necesidades nutricionales propias de cada etapa, y es a través de la alimentación como se puede cimentar bases para una vida sana y armónica (FAO, 2017), en los primeros años de vida se depende en totalidad del núcleo familiar, los padres son los encargados de los cuidados y la enseñanza de un sin fin de actividades que con el tiempo se convertirán en hábitos.

Así mismo el requerimiento energético del niño puede definirse como la ingesta calórica necesaria para mantener el funcionamiento óptimo de los órganos y sistemas que integran al cuerpo humano, entre las funciones corporales que más gasto energético tienen es el metabolismo basal aproximadamente entre el 50\%-60\% y en lo que respecta a la actividad física se le otorga un valor superior al 25\% (Vasudeman, Sreekumari, \& Vaydyanathan, 2011).

Gracias a las diferencias fisiológica y morfológica en comparación con otras especies, el hombre puede incluir una gran variedad de alimentos proporcionando los nutrientes necesarios para mantener la estructura y tejidos del organismo, la energía imprescindible para metabolismo corporal, para realizar la actividad física diaria y ser adecuada para que el niño desarrolle satisfactoriamente sus actividades escolares (Contreras \& Gracia, 2005).

La alimentación es un derecho humano universal que permite que las personas tengan acceso a una alimentación adecuada y a los recursos necesarios para tener en forma sostenible seguridad alimentaria. Este derecho representa no sólo un compromiso moral o una opción de políticas, sino que en la mayoría de los países constituye un deber de derechos humanos jurídicamente obligatorio de acuerdo a las normas internacionales de derechos humanos que han ratificado (Blanchi \& Szpak, 2016). 


\section{$\underline{\text { http://revistainvestigacionacademicasinfrontera.com }}$}

Gracias a la reforma constitucional pudo crearse la Ley para la Protección de los Derechos de Niñas, Niños y Adolescentes, la cual parte de que se debe proteger el ejercicio de los derechos humanos de niñas, niños y adolescentes, tanto en el ámbito público como privado (Diario Oficial de la Federación, 2014). Establece la obligación del Estado de proveer lo necesario para propiciar el respeto a la dignidad de la niñez y el ejercicio pleno de sus derechos, así como de otorgar facilidades a los particulares para que coadyuven al cumplimiento de los derechos de la niñez.

El estado nutricional, como resultado directo del consumo y la utilización de los alimentos, es un indicador importante del nivel de salud y de la calidad de vida de la población, así como del grado de satisfacción de sus necesidades básicas (Reyes \& Garduño, 2013).

En el estado nutricional se debe tener en cuenta la malnutrición y este término exige incluir tanto el déficit como el exceso de peso, ya que son estados fisiológicos que indican el estado de salud de la persona, mediante el cual se pueden tomar decisiones y abordar el problema de la mejor forma posible (Cañón et al, 2014).

Existes gran variedad de métodos para la valoración del estado nutricional lo que permitirá conocer el grado en que la alimentación cubre las necesidades del organismo, dicha evaluación debe ser un componente del examen rutinario en los niños (Morán, et al., 2015).

La valoración del estado nutricional es más que una disciplina, es un instrumento operacional, es decir un procedimiento que finalmente define conductas, con la información obtenida se puede estructurar y aplicar un plan de acción impactando positivamente. Por tanto la evaluación del estado nutricional será la acción y efecto de estimar, apreciar y calcular la condición en la que se encuentre un individuo según las modificaciones nutricionales que se hayan podido afectar (Figueroa, 2017). 


\section{$\underline{\text { http://revistainvestigacionacademicasinfrontera.com }}$}

La antropometría o cineantropometría fue presentada como una ciencia en 1976, en el Congreso Internacional de las Ciencias de la Actividad Física, celebrado en Montreal, y 2 años después fue aceptada como ciencia por la UNESCO, en el International Council of Sport and Physical Education (Carmenate, Moncada, \& Borjas, 2014).

La antropometría es un método doblemente indirecto que a través de mediciones del tamaño corporal, el peso y las proporciones, los cuales constituyen indicadores sensibles de la salud, desarrollo y crecimiento en el caso de los niños y jóvenes, de hecho es constituyente indispensable en la evaluación del estado nutricio de los individuos en cualquiera de los extremos de mala nutrición, tanto en la obesidad como la emaciación (Nariño, Becerra \& Hernández, 2016).

En la actualidad es uno de los métodos más utilizados para evaluar y vigilar el estado de salud, el estado nutricional y el crecimiento de los niños, tanto en individuos, como las comunidades, y está presente no solo en el are clínica, cuenta con un lugar importante en las denominadas ciencias que contribuyen en la formación de los docentes de la cultura física y deporte, son de fácil aplicación, bajo costo y reproducibilidad en diferentes momentos y con distintas personas (Ravasco, Anderson, \& Mardones, 2010).

Aparentemente la evaluación del estado nutricional mediante antropometría puede ser sencilla, pero se tiene que tener una capacitación previa, practica y llegar a la especialización, el dominio es fundamental para que los datos sean precisos y veraces (Carmina et al, 2016).

Como se menciona anteriormente el crecimiento y desarrollo guarda una estrecha relación entre la normalidad de las anteriores y el estado de salud de la persona, la antropometría es un indicador objetivo y tiene como propósito cuantificar la variación en las dimensiones físicas y la composición del cuerpo humano en diferentes edades (Montesinos-Correa, 2014). 


\section{$\underline{\text { http://revistainvestigacionacademicasinfrontera.com }}$}

Para la etapa escolar, se han revisado recientemente las curvas de crecimiento adaptándolas a las características de niños sanos desarrollados en ambientes saludables para obtener estándares antropométricos de referencia que reflejen mejor el crecimiento óptimo de los niños (Arija, et al, 2015).

Para el órgano internacional UNICEF, (2012) la aplicación de esta nueva referencia contribuye a la evaluación de las prácticas locales destinadas a la promoción del crecimiento y desarrollo y a la optimización de los recursos disponibles para mejorar el estado nutricional de los niños y niñas.

A partir de las medidas antropométricas se calculan diferentes índices que las relacionan y permiten valorar el estado nutricional, ya que las medidas por sí solas no conducen a valoraciones.

Las ventajas de los indicadores antropométricos entre otros son: a) no invasivos, b) accesible y fáciles de ejecutar, c) equipo barato para realizarlo. Entre sus desventajas podemos citar: a) requieren de personal capacitado, b) su confiabilidad depende de la precisión y exactitud (Castillo \& Zenteno, 2004). Los índices básicos para la etapa escolar son: Peso para la edad (P/E), Talla para la edad (T/E), Peso para la talla (P/T), Índice de masa corporal para la edad (IMC/E).

A pesar de los avances en materia de desnutrición infantil que se han experimentado en los últimos años, lo cierto es que las cifras siguen siendo alarmantes en algunos sectores de la población. En el grupo de edad de cinco a catorce años la desnutrición crónica es de $7.25 \%$ en las poblaciones urbanas, y la cifra se duplica en las rurales (UNICEF, 2017).

La desnutrición conduce a un estado de salud deficiente, esta favorece directamente a la enfermedad ya que tiene un efecto negativo en el sistema inmunológico, por lo tanto facilita los agentes patógenos colonicen el organismo, lo que a su vez aumenta propensión a sufrir enfermedades infecciosas y crónicas (Martínez, 2017). 


\section{$\underline{\text { http://revistainvestigacionacademicasinfrontera.com }}$}

La otra cara de los problemas de nutrición lo conforma la obesidad infantil, que ha ido creciendo de forma alarmante en los últimos años. Actualmente, México ocupa el primer lugar mundial en obesidad infantil, y el segundo en obesidad en adultos, precedido sólo por los Estados Unidos.

Se desconoce la incidencia del estado nutricional de los estudiantes inscritos en el mencionado centro educativo y teniendo en cuenta, que es trabajo de todos contribuir al adecuado crecimiento y desarrollo del infante, la promoción de alimentos saludables y la educación de calidad. Es de gran importancia la colaboración del personal docente y la sensibilización de los padres de familias para fortalecer estos factores para asegurar la vigilancia del estado nutricional, hecho que nos motiva a realizar el presente estudio en este centro escolar.

Por lo argumentado con anterioridad la valoración del estado nutricional y los cambios que se pueden dar en el mismo tanto en lo personal como colectivo se convierte en punto prioritario para todos los inmiscuidos en los cuidados de los niños (Enfermeros, pediatras, educadoras, docentes de la cultura física etc.) esta valiosa información es el punto de partida para el diseño de estrategias de intervención para afrontar la problemática en los polos del espectro nutricio.

\section{Formulación del problema}

¿Cuál es el estado nutricional de los estudiantes de la escuela primaria Niños Héroes del Municipio de Etchojoa, Sonora?

\section{Objetivo general}

Diagnosticar el estado nutricional en escolares de ambos sexos de nivel primaria $\left(1^{\mathrm{o}}\right.$ a $6^{\mathrm{o}}$ grado) de la Niños Héroes del Municipio de Etchojoa mediante el uso de indicadores antropométricos acordes a la edad. 


\section{http://revistainvestigacionacademicasinfrontera.com}

\section{Objetivos específicos}

- Evaluación de la estatura erguida de los estudiantes de 6-12 años de la escuela primaria Niños Héroes.

- Evaluación del peso de los estudiantes de 6-12 años de la escuela primaria Niños Héroes.

- Graficar en percentiles los indicadores (P/E, T/E, IMC/E) de la Organización Mundial de la Salud (OMS).

\section{Materiales y Métodos}

La presente es un estudio que recae en la tipología de investigación aplicada en campo, tiene como finalidad recoger y registrar ordenadamente los datos relativos al tema elegido como objeto de estudio (Baena, 2014) y descriptivo de corte trasversal ya que se intentan analizar el objeto de estudio en un periodo de tiempo corto, en otras palabras un punto en el tiempo, por eso también se les denomina "de corte" (Salinero, 2004), el diseño es no experimental ya que se realiza sin manipular deliberadamente variables. Se basa fundamentalmente en la observación de fenómenos tal y como se dan en su contexto natural para después analizarlo (Ruiz, 2012).

\section{Población y muestra}

Se estudiaron $n=48(92.3 \%)$ de 52 estudiantes de ambos sexos con rango de edad de 6 a 11 años de todos los grados y grupos de la escuela primaria pública Niños Héroes del Municipio de Etchojoa Sonora México, inscritos en el ciclo escolar 2017-1. El muestreo es no probabilístico intencional o por conveniencia, se elige ya que la matrícula inscrita en el ciclo mencionado es pequeña, se realiza un barrido en toda la matrícula de los grados $\left(1^{\mathrm{o}}\right.$ al $\left.6^{\mathrm{o}}\right)$. 


\section{$\underline{\text { http://revistainvestigacionacademicasinfrontera.com }}$}

\section{Criterios de inclusión}

Por ser una muestra no probabilística, la elección de los sujetos de estudio dependió de criterios de inclusión, estos se definen las principales características de la población y adquieren un carácter de gran importancia para la presente. Para su elección, se analizaron y consensaron de forma colectiva, quedando los siguientes:

a) Alumnos de ambos sexos pertenecientes a todos los grados $\left(1^{\circ}\right.$ al $\left.6^{\circ}\right)$ con rango 6 a 11 años.

b) Estar inscrito de forma oficial en el ciclo escolar 2017-1.

c) Autorización escrita por parte de los padres de familia o tutor con mayoría de edad.

d) Participar en la plática informativa sobre los objetivos y metodología de trabajo.

e) No presentar edema.

\section{Materiales}

Se utilizó un formato personal impreso de registro, en el cual se plasman dos apartados:

a) Datos sociodemográficos personales como: Nombre, edad, sexo, grado escolar.

b) Parámetros antropométricos: Peso y talla, con estos dos más la edad se determinó el Índice de Masa Corporal para la Edad (IMC/E).

Se realizó una prueba piloto para valorar la eficacia de los instrumentos de recolección de datos para poder detectar las posibles dificultades que se puedan presentar en el trabajo de campo, una vez comprobada la pertinencia tanto de la metodología como el instrumento, se aplicó a la unidad de análisis seleccionada para el estudio y para certificar la validez de los mismos.

Posteriormente al consentimiento escrito por el director de la institución educativa. La obtención de los datos se realizó en un área debidamente adaptada para la aplicación del 


\section{$\underline{\text { http://revistainvestigacionacademicasinfrontera.com }}$}

protocolo, cuenta con iluminación, modificación de temperatura, higiene, lo principal resguardaba la privacidad y seguridad de cada alumno.

El protocolo de medición antropométrico es basado en las recomendaciones de la Sociedad Internacional para el Avance de la Cineantropometría (ISAK) por sus siglas en Ingles (ISAK, 2001). Las medias antropométricas y anotaciones se realizaron por los autores de la presente.

Se estudiaron los indicadores de masa corporal por percentil tales como: la talla para la edad (TE), el peso para la edad (PE) y el Índice de Masa Corporal para la Edad (IMC/E).

La actuación tras el análisis de los datos; una vez recopilados toda la información de las mediciones antropométricas y verificada su valoración, se remitió a los padres de familia, a través de la dirección del centro escolar, una carta informe sobre el resultado del estado nutricional de su hijo(a), proporcionando consejo al respecto, en función de los resultados obtenidos.

\section{Consideraciones éticas}

Los autores López-Pacheco, Pimentel-Hernández, Rivas-Mirelles, \& Arredondo-García, (2016) argumenta que para realización de cualquier estudio clínico, sin importar la fase de investigación en la que se encuentre, ya sea para el empleo de insumos para la salud, procedimientos o actividades experimentales en seres humanos o muestras biológicas de seres humanos, deben respetarse los principios científicos, éticos y regulatorios nacional e internacionalmente aceptados. Para esto, el anteproyecto de Tesis es revisado y avalado por la junta de academia de la Licenciatura en Entrenamiento Deportivo de la Universidad Estatal de Sonora.

El estudio se llevó acabo siguiendo las Normas Deontológicas reconocidas por la Declaración de Helsinki de 1975 (AMM, 2013), de igual forma se acogió a la Norma Oficial Mexicana NOM-012-SSA3-2012 que establece los criterios para la ejecución de 


\section{$\underline{\text { http://revistainvestigacionacademicasinfrontera.com }}$}

proyectos de investigación para la salud en seres humanos (Secretaría de Salud, 2013), y se ajustó a los principios científicos y éticos de la Ley General de Salud (Ley General de Salud , 2017), en la cual destacan los siguientes artículos

Artículo 96.- La investigación para la salud comprende el desarrollo de acciones que contribuyan la prevención y control de los problemas de salud que se consideren prioritarios para la población.

Artículo 100.- que señala que la investigación en seres humanos se desarrollará sólo cuando exista una razonable seguridad de que no expone a riesgos ni daños innecesarios al sujeto en experimentación, se deberá contar con el consentimiento informado por escrito del sujeto en quien se realizará la investigación.

\section{Análisis estadístico}

Como primer paso se crea una base de datos en hoja de Cálculo Microsoft Excel 2013, posteriormente para la graficaciòn de las variables antropométricas y con el objetivo de obtener el diagnóstico nutricional se utilizó el Sofwer Anthro Plus v 1.0.4, desarrollado para la aplicación de la referencia más actuales de la OMS para los niños y adolescentes de 6-11 años.

Con este paso se llevó a cabo un análisis exploratorio de los datos, donde la estadística descriptiva de las mediaciones antropométricas fueron analizadas a través de medidas de tendencia central estratificadas por género y rangos de edad, se expresan en valor de media $\dot{X}) \pm$ la desviación estándar (DE), número, porcentaje, máximos y mínimos, para el contraste de normalidad se aplica la prueba de Shapiro-Wilk, se utilizó el paquete Statical Package for Social Sciences® (SPSS V.21) para Windows.

\section{Resultados}

Se presentan a continuación los resultados obtenidos del trabajo de campo de la investigación aplicada en la escuela primaria Niños Héroes del Municipio de Etchojoa, 


\section{$\underline{\text { http://revistainvestigacionacademicasinfrontera.com }}$}

en la cual se encuentran inscritos un total de 52 alumnos que asisten regularmente a al turno vespertino, tras la depuración basándonos en los criterios de inclusión, se estudiaron 48(92.3\%) de los cuales el 19(39.5\%) pertenece al sexo masculino predominando en el sexo femenino $29(60.4 \%)$.

\section{Tabla 1}

Distribución de la población por edad y sexo

\begin{tabular}{ccccccc}
\hline $\begin{array}{c}\text { Edad } \\
\text { (años) }\end{array}$ & \multicolumn{2}{c}{ Hombres } & \multicolumn{2}{c}{ Mujeres } & \multicolumn{2}{c}{ Total } \\
\cline { 2 - 7 } & $N$ & $\%$ & $n$ & $\%$ & $n$ & $\%$ \\
7 & 6 & 66.6 & 3 & 33.3 & 9 & 18.7 \\
8 & 2 & 33.3 & 4 & 66.6 & 6 & 12.5 \\
9 & 1 & 14.2 & 6 & 85.7 & 7 & 14.5 \\
10 & 2 & 33.3 & 4 & 66.6 & 6 & 12.5 \\
11 & 2 & 20.0 & 8 & 80.0 & 10 & 20.8 \\
Total & 6 & 60.0 & 4 & 40.0 & 10 & 20.8 \\
\hline & $\mathbf{1 9}$ & $\mathbf{3 9 . 5}$ & $\mathbf{2 9}$ & $\mathbf{6 0 . 4}$ & $\mathbf{4 8}$ & $\mathbf{1 0 0}$
\end{tabular}

El rango de edad de los alumnos estudiados oscila de entre los 6 y 11 años con una media

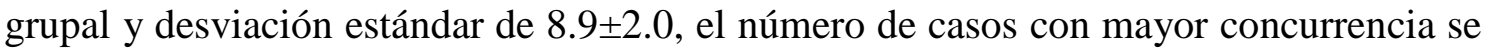
encuentran en las edades comprendidas de 6,10 y 11 años, dando un número de casos y similar 10(20.8\%), en contra parte, en la edad donde menos casos es en las edades de 7 y 9 años $6(12.5 \%)$.

En lo que respecta a la variable peso de forma grupal cuenta con un promedio de 29.9 con desviación estándar de 6.9, teniendo una ligera elevación en el sexo femenino 29.47 7.03 en comparación con $28.82 \pm 6.95$ para el sexo masculino.

En la tabla 1 de igual forma, se plasma los resultados de la misma variable con fragmentación por edad tanto para hombres como mujeres, teniendo valores menores en la edades distales a la etapa puberal, en el la edad de 6 años sexo masculino se aprecia que el peso cuenta con una media y desviación estándar de $21.7 \pm 2.4$, respecto al sexo femenino presentan valores muy similares solo varia por decimales en la media 21.3(DE=0.6), en las edades mayores en específico 11 años de edad sexo masculino 


\section{$\underline{\text { http://revistainvestigacionacademicasinfrontera.com }}$}

encontramos que en el peso se obtiene un valor medio de 33.6(DE=5.1) y el valor medio mayor en el sexo femenino con 40.0(DE=9.6).

Tabla 2

Media, desviación estándar, mínimo y máximo de los variables peso y talla en ambos sexos

\begin{tabular}{|c|c|c|c|c|c|c|c|}
\hline \multirow[t]{4}{*}{ Variable } & \multirow{2}{*}{$\begin{array}{c}\text { Edad } \\
\text { (Años) }\end{array}$} & \multicolumn{3}{|c|}{ Hombres } & \multicolumn{3}{|c|}{ Mujeres } \\
\hline & & $X \pm D E$ & Mín & Máx & $X \pm D E$ & Mín & Máx \\
\hline & 6 & $21.7 \pm 2.4$ & 19.5 & 25.2 & $21.3 \pm 0.6$ & 20.0 & 22.1 \\
\hline & 7 & $27.1 \pm 8.4$ & 21.1 & 33.0 & $24.6 \pm 2.9$ & 21.5 & 28.2 \\
\hline \multirow[t]{7}{*}{ Peso (Kg) } & 8 & - & - & - & $30.7 \pm 3.2$ & 27.5 & 36.5 \\
\hline & 9 & $32.6 \pm 6.5$ & 27.9 & 37.1 & $30.5 \pm 4,2$ & 25.1 & 35.1 \\
\hline & 10 & $34.8 \pm 5.9$ & 30.6 & 39.1 & $28.2 \pm 5.3$ & 21.2 & 38.5 \\
\hline & 11 & $33.6 \pm 5.1$ & 28.9 & 43.6 & $40.0 \pm 9.6$ & 31.3 & 53.5 \\
\hline & 6 & $110.3 \pm 3.1$ & 106.0 & 114.9 & $108.6 \pm 0.5$ & 108.0 & 109.1 \\
\hline & 7 & $116.2 \pm 9.8$ & 109.3 & 123.2 & $112.0 \pm 4.1$ & 107.1 & 117.0 \\
\hline & 8 & - & - & - & $122.5 \pm 5.1$ & 117.6 & 130.3 \\
\hline \multirow[t]{3}{*}{ Talla (cm) } & 9 & $131.2 \pm 1.4$ & 130.2 & 132.2 & $120.1 \pm 6.3$ & 115.2 & 129.3 \\
\hline & 10 & $129.0 \pm 9.8$ & 122.1 & 136.0 & $123.3 \pm 4.0$ & 115.7 & 128.0 \\
\hline & 11 & $127.2 \pm 6.0$ & 120.0 & 137.1 & $135.6 \pm 5.6$ & 130.2 & 141.0 \\
\hline
\end{tabular}

Nota: $\mathrm{X}=$ media, $\mathrm{DS}=$ desviación estándar

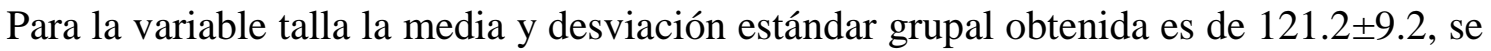
obtiene una estatura media similar en ambos sexos con una pequeña diferencia por décimas, en el sexo femenino $121.33 \pm 8.9$ en comparación con los valores del sexo

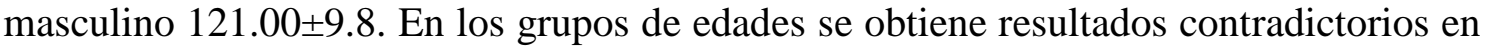
la variable anteriormente mencionada ya que en la edad de 9 años se alcanza una media y desviación estándar de $131.2 \pm 1.4$ en el sexo masculino, resultado mayor a sus

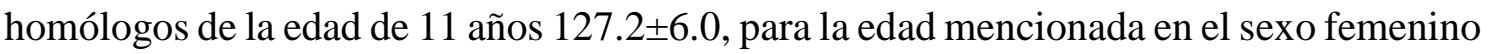
forma se obtienen valores medios mayores $135.6(\mathrm{DE}=5.6)$.

En el indicador peso para la edad aplicado en las edades comprendidas de los 6 a los 9 años, 26(92.8\%) se encuentran entre los percentiles 10 al 90 indicadores de normalidad en el peso y adecuado estado nutricional con una media y desviación estándar de 


\section{$\underline{\text { http://revistainvestigacionacademicasinfrontera.com }}$}

62.3 \pm 21.7 , en los percentiles bajos que indican desnutrición crónica no se encontraron casos y dos de los casos restantes se encuentran en sobre peso p90-97 y obesidad p $>97$.

El 9(90\%) del alumnado del sexo masculino se posiciona entre los percentiles 10-90 con media y desviación estándar de 58.7 25.5 indicando un peso y estado nutricional normal, solo un caso cuenta con sobre peso p90-97 resultado similar al sexo femenino ya que $15(93.7 \%)$ se encuentra en los percentiles de rango medio 90-97 y solo un caso en $>97$ por ende obesidad, la media de los percentiles es de 64.7(DE=19.8).

Tabla 3

Valoración antropométrica mediante el indicador peso para la edad en ambos sexos

Percentil

\begin{tabular}{cccccccccc}
\hline $\begin{array}{c}\text { Edad } \\
\text { (Años) }\end{array}$ & Sexo & $X \pm D E$ & Mín & Máx & $<3$ & $3-10$ & $10-90$ & $90-97$ & $>97$ \\
\hline \multirow{2}{*}{6} & H & $56.9 \pm 24.1$ & 29.9 & 86.4 & 0 & 0 & 6 & 0 & 0 \\
& M & $62.8 \pm 4.2$ & 58.8 & 67.1 & 0 & 0 & 3 & 0 & 0 \\
7 & H & $64.3 \pm 45.0$ & 32.4 & 96.1 & 0 & 0 & 1 & 1 & 0 \\
& M & $56.1 \pm 23.5$ & 34.1 & 84.7 & 0 & 0 & 4 & 0 & 0 \\
$\mathbf{8}$ & H & -------- & 41.2 & ---- & 0 & 0 & 1 & 0 & 0 \\
& M & $77.7 \pm 16.0$ & 51.5 & 97.6 & 0 & 0 & 5 & 0 & 1 \\
$\mathbf{9}$ & H & $67.0 \pm 27.6$ & 47.5 & 86.5 & 0 & 0 & 2 & 0 & 0 \\
& M & $55.4 \pm 23.2$ & 22.6 & 77.1 & 0 & 0 & 4 & 0 & 0 \\
\hline
\end{tabular}

Nota: X media DS desviación estándar

En la Tabla 4 se muestra la distribución de los escolares por percentil del indicador talla para la edad en todos los grupos de edades y ambos sexos, se obtiene una media de los sujetos evaluados de 9.9(DE=15.3), en el sexo masculino encontramos una media y

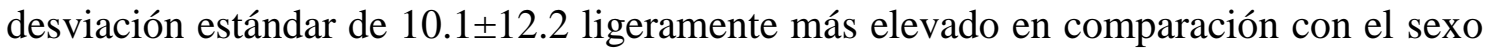
masculino obteniendo los valores $9.8 \pm 17.2$.

El 27.8\%(n=13) del total de los escolares estudiados se encuentra en una talla adecuada para su edad, ya que se posicionan en los percentiles 10 al 90, de los cuales los hombres representan el $14.5 \%(n=7)$ y el $12.5 \%(n=6)$ para el sexo femenino. Por otra parte en el aspecto que indica problemas de retraso de crecimiento o talla baja para la edad, encontramos que el $18.7 \%(n=9)$ se encuentran en la zona de riesgo de los cuales destaca 


\section{$\underline{\text { http://revistainvestigacionacademicasinfrontera.com }}$}

el sexo femenino con el $8.3 \%(n=4)$, el resto de los escolares se encuentran con problemas de talla baja $54.1 \%(n=26)$.

Tabla 4

Valoración antropométrica mediante el indicador talla para la edad en ambos sexos

Percentil

\begin{tabular}{cccccccccc}
\hline $\begin{array}{c}\text { Edad } \\
\text { (Años) }\end{array}$ & Sexo & $\boldsymbol{X} \pm \boldsymbol{D E}$ & Mín & Máx & $<\mathbf{3}$ & $\mathbf{3 - 1 0}$ & $\mathbf{1 0 - 9 0}$ & $\mathbf{9 0 - 9 7}$ & $>\mathbf{9 7}$ \\
\hline \multirow{2}{*}{$\mathbf{6}$} & H & $13.3 \pm 13.9$ & 2.3 & 37.8 & 2 & 1 & 3 & 0 & 0 \\
& M & $9.3 \pm 2.4$ & 7.8 & 12.0 & 0 & 2 & 1 & 0 & 0 \\
$\mathbf{7}$ & H & $13.6 \pm 17.2$ & 1.4 & 25.7 & 1 & 0 & 1 & 0 & 0 \\
& M & $3.6 \pm 4.9$ & 0.4 & 10.9 & 3 & 0 & 1 & 0 & 0 \\
$\mathbf{8}$ & H & ------- & 3.7 & ------- & 0 & 1 & 0 & 0 & 0 \\
& M & $24.5 \pm 29.1$ & 1.3 & 97.6 & 1 & 2 & 3 & 0 & 0 \\
$\mathbf{9}$ & H & $27.6 \pm 7.9$ & 22 & 33.2 & 0 & 0 & 2 & 0 & 0 \\
& M & $7.4 \pm 14.4$ & 0.1 & 29 & 3 & 0 & 1 & 0 & 0 \\
$\mathbf{1 0}$ & H & $8.2 \pm 11.4$ & 0.1 & 16.2 & 1 & 0 & 1 & 0 & 0 \\
& M & $0.6 \pm 0.6$ & 0.1 & 1.7 & 8 & 0 & 0 & 0 & 0 \\
& H & $1.5 \pm 2.4$ & 0.1 & 6.1 & 5 & 1 & 0 & 0 & 0 \\
& M & $15.1 \pm 19.1$ & 0.3 & 41 & 2 & 2 & 0 & 0 & 0 \\
\hline
\end{tabular}

Nota: $X=$ media, $D S=$ desviación estándar

El índice de masa corporal para la edad se representa a manera de percentil en la tabla 5 , el $37.5 \%(n=18)$ cuenta con un estado nutricional adecuado, en los percentiles que indican sobre peso se encuentran el 39.5\%(n=19) del total de los integrantes de la mencionada institución educativa, el 20.8\%(n=10) de los mencionados pertenecen al sexo masculino.

Tabla 5

Valoración del estado nutricional mediante el indicador IMC/E para la edad en ambos sexos

\begin{tabular}{ccccccccccc}
\hline $\begin{array}{c}\text { Edad } \\
\text { (Años) }\end{array}$ & Sexo & X \pm DE & Mínimo & Máximo & $<\mathbf{3}$ & $\mathbf{3 - 1 0}$ & $\mathbf{1 0 - 9 0}$ & $\mathbf{9 0 - 9 7}$ & $>\mathbf{9 7}$ \\
\hline \multirow{6}{*}{} & Hombres & $89.4 \pm 10.5$ & 70.1 & 99.8 & 0 & 0 & 2 & 3 & 1 \\
& Mujeres & $93.2 \pm 2.0$ & 96.1 & 95.4 & 0 & 0 & 0 & 3 & 0 \\
\multirow{2}{7}{} & Hombres & $95.8 \pm 5.5$ & 91.9 & 99.7 & 0 & 0 & 0 & 1 & 1 \\
& Mujeres & $95.0 \pm 4.5$ & 88.9 & 98.5 & 0 & 0 & 0 & 2 & 2 \\
$\mathbf{8}$ & Hombres & ------- & 88.5 & ------ & 0 & 0 & 1 & 0 & 0 \\
\hline
\end{tabular}


http://revistainvestigacionacademicasinfrontera.com

\begin{tabular}{cccccccccc}
\hline & Mujeres & $96.3 \pm 2.0$ & 93.4 & 98.7 & 0 & 0 & 0 & 4 & 2 \\
$\mathbf{9}$ & Hombres & $79.0 \pm 26.4$ & 60.3 & 90.7 & 0 & 0 & 1 & 1 & 0 \\
& Mujeres & $80.7 \pm 36.4$ & 26.1 & 99.5 & 0 & 0 & 1 & 0 & 3 \\
\multirow{110}{*}{} & Hombres & $94.2 \pm 1.6$ & 93 & 95.3 & 0 & 0 & 0 & 2 & 0 \\
& Mujeres & $63.9 \pm 28.4$ & 22.3 & 99.1 & 0 & 0 & 7 & 0 & 1 \\
\multirow{11}{*}{} & Hombres & $89.0 \pm 8.2$ & 75.7 & 97.5 & 0 & 0 & 3 & 3 & 0 \\
& Mujeres & $83.5 \pm 19.1$ & 55.8 & 99.5 & 0 & 0 & 3 & 0 & 1 \\
\hline
\end{tabular}

Nota: X media DS desviación estándar

Por ultimo en el percentil $>97$ el cual indica obesidad el 22.9\%(n=11) presentan malnutrición por exceso destacando el sexo femenino con $18.7 \%(n=9)$ del total, por otra parte tanto en la zona de riesgo y bajo peso no encontramos casos siguiendo el indicador antes mencionado.

\section{Discusión}

Es notoria la baja matricula que integra a la escuela Niños Héroes la cual se encuentra ubicada en la comunidad de Bacobampo, esto pude presentarse por dos factores: 1) la comunidad cuenta con una población total 8011 personas (cantidad baja en comparación con otras del mismo estado, 2)- Existen 216 analfabetos de 15 y más años, 32 de los jóvenes entre 6 y 14 años no asisten a la escuela.

Para la valoración del estado nutricional en las edades de 6 a 9 años, la organización mundial de la salud (OMS) recomienda este indicador, los estándares de peso para la edad no están disponibles para los niños de más de 10 años. La razón es el peso para la edad en niños mayores de 10 años no permite distinguir exceso de talla de exceso de masa corporal. Durante este periodo de la pubertad los niños pueden aparecer obesos (usando peso para la edad) cuando solamente están creciendo en talla.

Es muy posible que la baja talla no sea más que una manifestación de la existencia de condiciones alimenticias inadecuadas, que actúan en la población objeto de estudio ya 


\section{$\underline{\text { http://revistainvestigacionacademicasinfrontera.com }}$}

que el municipio y localidad donde se ubica la institución educativa es catalogado como de extrema pobreza sumado al bajo nivel académico.

En el estado de Sonora el total de alumnado debidamente matriculado en el sistema educativo público en educación primaria es de 285,014, el sexo masculino cuenta con mayor presencia con 145,541 estudiantes, en comparación al sexo femenino el cual cuenta con 139,473 estudiantes (SEP, 2016).

Los resultados del estado nutricional de la investigación tienen similitud con los de ENSANUT 2016, en la mencionada encuesta la prevalencia combinada de sobrepeso y obesidad en la población en edad escolar fue 33.2\%, así mismo en el estado de Sonora el 26.9 por ciento de los niños entre los 5 y 11 cuentan con esta problemática.

En el estudio regional de Castañeda-Sánchez, Lugo-Caro, \& Yepiz, (2016) de igual formorma encontramos numeros alarmantes respecto al sobrepeso y obesidad, pese a que la poblacion tiene un rango de edad mayor es aplicado en un poblado con caracteristicas similares.

\section{Conclusiones y recomendaciones}

Si bien, el objetivo general es la Valoración del estado nutricional mediante un método doblemente indirecto como lo es la antropometría se determina que la un porcentaje mayor de la población en general cuenta con un estado nutricional adecuado en los indicadores peso para la talla, talla para a edad y el IMC para la edad. Por otra parte no se puede dejar de lado los porcentajes ubicados en las zonas de malnutrición, ya que encontramos que coexisten casos de mal nutrición en los dos espectros.

Según Torres, Duque, Granada, Serna \& García (2015) los problemas de malnutrición que no se detectan precoz mente puede comprometer la salud del infante, y es propenso a transpolar el problema a futuras etapas de la vida, 


\section{$\underline{\text { http://revistainvestigacionacademicasinfrontera.com }}$}

Por lo cual la importancia de la valoración del estado nutricional en edades tempranas, la información obtenida adquiere un carácter preventivo, predictivo y de orientación para el trabajo con la persona, en esta misma línea es primordial capacitar y concientizar a todo el personal inmiscuido con los cuidados de los niños.

Los datos obtenidos en la investigación nos compromete a concientizar a los padres respecto a los hábitos de Vida Saludable haciendo énfasis en la actividad física y una alimentación adecuada, queda de manifiesto a lo largo de nuestro aporte, la suma importancia de los dos aspectos anteriormente mencionados, esto con el fin de coadyuvar al sano y adecuado crecimiento y desarrollo del niño. Por otro lado será imposible sin el apoyo de factores tales como: familia, escuela sociedad y autoridades gubernamentales. Otro aspecto que se puede plantear es la aplicación de una intervención para el tratamiento y mejora de los casos que presenten malnutrición.

\section{Bibliografía}

Ulíbarri, I., Lobo, G., \& Pérez, J. (2015). Desnutrición clínica. Nutr Clin Med, IX(3), 231-254. doi:DOI: 10.7400/NCM.2015.09.3.5033

AMM. (Octubre de 2013). Declaración de Helsinki de la AMM Principios éticos para las investigaciones médicas en seres humanos. Recuperado el 23 de 01 de 2017, de https://www.wma.net/es/policies-post/declaracion-de-helsinki-de-la-amm-principioseticos-para-las-investigaciones-medicas-en-seres-humanos/

Aranceta, J., Perèz, C., \& Garcìa, M. (2002). Nutricion Comunitaria. Santander: Servicio de Publicaciones de la Universidad de Cantabria.

Arija, V., Pérez, C., Martínez, E., Ortega, R. M., Serra-Majem, L., Ribas, L., \& Aranceta, J. (2015). Valores de referencia de ingesta dietética y de antropometría en estudios poblacionales. Rev Esp Nutr Comunitaria, 21(1), 157-167. doi:DOI: 10.14642/RENC.2015.21.sup1.5062

Baena, G. (2014). Metodologia de la Investigacion. Mexico, D F: Patria.

Blanchi, E., \& Szpak, C. (2016). Seguridad Alimentaria y el Derecho a la Alimentación Adecuada. Revista Brasileira de Estudos Jurídicos, 37-45. 


\section{http://revistainvestigacionacademicasinfrontera.com}

Cañizares, J. M., \& Carbonero, C. (2017). Crecimiento y desarrollo del niño. Sevilla, España: Wamceulen.

Cañón, S. C., Castaño , J. J., Duque, D. C., Llano, V., Martínez, I., Méndez, L., . . Ramírez, W. A. (2014). Estudio multifactorial del estado nutricional y comparación tablas OMS colombianas en niños de primero de primaria de 6 instituciones educativas de la ciudad de Manizales 2012-2014. Archivos de Medicina, 14(2), 236-348.

Carmenate, L., Moncada, F. A., \& Borjas, E. W. (Mayo de 2014). MANUAL DE MEDIDAS ANTROPOMÉTRICAS. Heredia, Costa Rica : Publicaciones SALTRA. Obtenido de http://www.repositorio.una.ac.cr/bitstream/handle/11056/8632/MANUAL\%20ANTRO POMETRIA.pdf? sequence $=1$

Carmina, M. A., de Mateo, B., Malafarina, V., Lopez, R., Niño, V., Lòpez, J. A., \& Redondo, M. P. (2016). Valoración del estado nutricional en Geriatría: declaración de consenso del Grupo de Nutrición de la Sociedad Española de Geriatría y Gerontología. Revista Española de Geriatría y Gerontología, 51(1), 52-57. doi:https://doi.org/10.1016/j.regg.2015.07.007

Castañeda-Sánchez, O., Lugo-Caro, M., \& Yepiz, R. (2016). Estado nutricional en un grupo de adolescentes de Pueblo Yaqui, Sonora, México. Atención Familiar, 23(3), 104-108. doi:https://doi.org/10.1016/j.af.2016.07.004

Castillo, J. L., \& Zenteno, R. (2004). Valoración del Estado Nutricional. Revista Médica de la Universidad Veracruzana, 4(2), 29-35.

Contreras , J., \& Gracia, M. (2005). Alimentación y cultura: perspectivas antropológicas. Barcelona : Ariel.

Courtois, V. R. (2014). Situación nutricional en escolares de Santiago el Pinar, Chiapas. Tesis para la obtencion de grado de Doctor. Universidad Complutense de Madrid. Madrid, España.

Diario Oficial de la Federación. (04 de 11 de 2014). LEY GENERAL DE LOS DERECHOS DE NINNAS, NINOSOS Y ADOLESCENTES. Obtenido de http://www.diputados.gob.mx/LeyesBiblio/pdf/LGDNNA_230617.pdf

Erikson, E. H. (1950). Childhood and society. New York: Norton.

FAO. (05 de 01 de 2013). El estado mundial de la agricultura y la alimentacion. Obtenido de http://www.fao.org/docrep/018/i3301s/i3301s.pdf

FAO. (3 de 08 de 2017). NUTRICIÓN $Y$ SALUD. Obtenido de http://www.fao.org/docrep/013/am283s/am283s05.pdf

Farrè, R. (03 de 06 de 2017). Evaluación del estado nutricional (dieta, composición corporal, $\begin{array}{llll}\text { bioquímica } & y & \text { clínica }) & \text { Obtenido de }\end{array}$ 


\section{$\underline{\text { http://revistainvestigacionacademicasinfrontera.com }}$}

https://www.kelloggs.es/content/dam/newton/media/manual_de_nutricion_new/Manual _Nutricion_Kelloggs_Capitulo_07.pdf

Figueroa, G. (28 de 04 de 2017). Contenidos Teóricos: Evaluación Nutricional. Obtenido de http://www.fmed.uba.ar/depto/nutrievaluacion/2015/evaluacion.pdf

Gómez, F. (2003). Desnutrición. salud pública de méxico, 45(4), S576-S582.

Hurtado-Quintero, C., Mejía, C., Mejía, F., Arango, C., Chavarriaga, L. M., \& Grisales-Romero, H. (2017). Malnutrición por exceso y déficit en niños, niñas y adolescentes, Antioquia, 2015. Fac. Nac. Salud Pública, 35(1), 58-70. doi:10.17533/udea.rfnsp.v35n1a07

Ibarra, L. S., Viveros, L. S., González, V., \& Hernández, F. (2017). Transición Alimentaria en México. Razón y Palabra, 3(94), 166-182.

ISAK. (2001). Estándares internacionales para la valoración antropométrica. Australia: ISAK.

Kliegman, R. M., Staton, B. M., \& Geme, J. (2016). Nelson. Tratado de pediatría. Brasil: ELSERVER.

Ley General de Salud . (27 de 01 de 2017). Recuperado el 15 de 01 de 2017, de http://www.diputados.gob.mx/LeyesBiblio/pdf/142_270117.pdf

Martínez, R. (2017). Salud y enfermedad del niño y del adolescente. Ciudad de Mexxico: El manual moderno.

Montesinos-Correa, H. (2014). Crecimiento y antropometría: aplicación clínica. Acta Pediátr Mex, 35, 159-165.

Ng, M., Fleming , T., Robinson, M., Thomson, B., Graetzq, N., \& Margono, C. (2014). Global, regional, and national prevalence of overweight and obesity in children and adults during 1980-2013: a systematic analysis for the Global Burden of Disease Study 2013. Lancet, 384(9945), 766-781. doi:10.1016/S0140-6736(14)60460-8

OMS. (s.f.). Patrones de crecimiento infantil de la OMS. Recuperado el 26 de 04 de 2017, de http://www.who.int/childgrowth/standards/tr_summary_spanish_rev.pdf

Ortiz-Hernández, L., \& Pérez-Salgado, D. (2011). Estratificación socioeconómica y daños a la. Medicina Social,, ll(1), 13-17.

Peniche , C., \& Boulllosa , B. (2011). Nutriciòn aplicada al deporte. Mèxico D.F: Mc Graw Hill.

Perichart, O. (2013). Manual de lineamientos para la práctica de la nutrición clínica. Mexio D.F: McGraw-Hill International.

Piaget, J. (1969). El nacimiento de la inteligencia del niño. Madrid: Aguilar. 


\section{$\underline{\text { http://revistainvestigacionacademicasinfrontera.com }}$}

Pizzo, M. E. (11 de 07 de 2017). El desarrollo de los niños en edad escolar. Obtenido de http://23118.psi.uba.ar/academica/carrerasdegrado/psicologia/informacion_adicional/ob ligatorias/053_ninez1/files/el_desarrollo_de_los_ninos_en_edad_escolar.pdf

Ravasco, P., Anderson, H., \& Mardones, F. (2010). Métodos de valoración del estado nutricional. Nutrición Hospitalaria, 3(3), 57-66.

Reyes, E., \& Garduño, F. (2013). ESTADO NUTRICIONAL EN LOS ESTUDIANTES DE LA Escuela Primaria "Heriberto EnriqueZ" del Municipio de Toluca, Estado de Mexico, 2012. Tesis para obtener el grado medico de cirujano. Toluca, Estado de Mexico, Mexico: Universidad Autonoma del Estado de Mexico .

Rice, F. P. (1997). Desarrollo Humano: Estudio del Ciclo Vital. Estado de Mexico: Pretince-Hall Hispanoamericana.

Román, S., Ojeda-Granados, C., \& Panduro, A. (2013). Genética y evolución de la alimentación de la población en México. Revista de Endocrinología y Nutrición, 21(1), 42-51. Obtenido de http://www.medigraphic.com/pdfs/endoc/er-2013/er131f.pdf

Ruiz, J. I. (2012). Teoria y Practica de la investigacion cualitatitiva. Bilbao: Universidad de Deusto.

Salinero, J. G. (06 de 2004). Estudios Descriptivos. Recuperado el 26 de 09 de 2017, de http://webpersonal.uma.es/ jmpaez/websci/bloqueiii/docbiii/estudios\%20descriptivos.p df

Secretaría de Salud. (04 de 01 de 2013). Norma Oficial Mexicana NOM-012-SSA3-2012, Que establece los criterios para la ejecución de proyectos. Obtenido de http://www.cofepris.gob.mx/MJ/Documents/Normas/nom-012ssa3investigaci\%C3\%B3n.pdf

SEP. (10 de 2016). Estadisticas Del Sistema Educativo Sonora Ciclo Escolar 2015-2017. $\begin{array}{lllllll}\text { Recuperado el } & 8 & \text { de } & 12 & \text { de } & 2017, & \text { de }\end{array}$ http://www.snie.sep.gob.mx/descargas/estadistica_e_indicadores/estadistica_e_indicado res_educativos_26SON.pdf

Shamah-Levy, T., Cuevas-Nasu , 1., Rivera-Dommarco, J., \& Hernández-Ávila, M. (2016). Encuesta Nacional de Nutrición y Salud de Medio Camino 2016 (ENSANUT 2016). Obtenido de http://promocion.salud.gob.mx/dgps/descargas1/doctos_2016/ensanut_mc_2016310oct.pdf

Torres, L. E., Duque, J. A., Granada, J., Serna, M., \& García, R. A. (2015). Anomalías dentales y su relación con la malnutrición en la primera infancia: un análisis crítico de literatura. 11(20), 65-69. doi:http://dx.doi.org/10.16925/od.v11i20.941 


\section{$\underline{\text { http://revistainvestigacionacademicasinfrontera.com }}$}

UNICEF. (07 de 2012). Evaluación del crecimiento de niños y niñas. Recuperado el 02 de 08 de 2017, de https://www.unicef.org/argentina/spanish/Nutricion_24julio.pdf

UNICEF. (18 de 05 de 2017). Salud y nutrición. Obtenido de El doble reto de la malnutrición y la obesidad: https://www.unicef.org/mexico/spanish/17047.htm

Vasudeman, D. M., Sreekumari, S., \& Vaydyanathan, K. (2011). Texto de Bioquimica para Estudiantes de Medicina. Guadalajara, Jalisco, Mexico : Cuellar Ayala.

Villegas, D. (2012). Fisiopatología general de la nutrición. 4, 259-276.

whitney, E., \& Rady, S. (2011). Tratado general de la Nutriciòn. Mexico D.F: Paidotribo. 